\title{
Coherence Probe Microscopy Imaging and Analysis for Fiber-Reinforced Polymers
}

\author{
Verena Schlager $^{1}$, Stefan E. Schausberger ${ }^{2}$, David Stifter $^{2}$, and Bettina Heise ${ }^{1,2}$ \\ Christian Doppler Laboratory for Microscopic and Spectroscopic Material \\ Characterization, \\ ${ }^{1}$ Department of Knowledge-Based Mathematical Systems, FLLL \\ ${ }^{2}$ Center for Surface- and Nanoanalytics, ZONA \\ Johannes Kepler University Linz, A-4040 Linz, Austria \\ \{bettina.heise, verena.schlager\}@jku.at \\ http://www.jku.at
}

\begin{abstract}
The potential of full-field low coherence interferometric techniques for imaging internal structures, such as fibers, interfaces, or inclusions in technical materials is demonstrated by our coherence probe microscopy (CPM) setup. However, the huge amount of recorded data demand for an automatized enhancement and evaluation of the image data. We propose an automatic image analysis procedure adapted for full-field coherence probe microscopy, which we tested on fiber composite materials. The performed image enhancement and orientation analysis finally allow to cluster the internal fiber structures, to detect outliers and enable an improved characterization of investigated specimens supporting a sophisticated material design for the future.
\end{abstract}

Keywords: coherence probe microscopy, fiber composites, speckles, orientation, monogenic, clustering.

\section{Introduction}

The increasing demand of industry for new functional materials requires appropriate methods for material characterization and inspection. Aggregates and composite materials containing structures in the size range of few microns altering material behavior are of crucial interest in the field of material design. Microscopy techniques on micrograph sections provide excellent conditions for the analysis of internal microstructures for further material characterization. But, these methods are hampered by the destructive character of this investigation techniques. Hence, non-destructive imaging techniques in combination with an appropriate image analysis for deepening the knowledge about the material composition on a mesoscopic size scale are on the order of the day.

Computer tomography (CT) and ultrasound (US) imaging are well known non-destructive imaging techniques. However, they are restricted in their application due to the hazardous X-ray radiation and the achievable resolution limited by the relation to the sample dimensions, or as in case of US by requiring an

A. Heyden and F. Kahl (Eds.): SCIA 2011, LNCS 6688, pp. 424 434, 2011.

(C) Springer-Verlag Berlin Heidelberg 2011 
additional coupling medium. For investigation of translucent or scattering materials low coherence interferometry (LCI) techniques working in the near infrared wavelength range (NIR) can provide an alternative to CT methods. Their imaging capabilities can be tuned for the visualization of micro-structured materials. Especially the characterization of the internal structure of the specimen within semi-transparent scattering materials can be achieved by optical coherence tomography (OCT) techniques 1, having its origin in LCI techniques. Exploiting both intensity and run-time respectively phase information of the backscattered signal depth-resolved information about e.g. the matrix pattern, disturbances or inclusions inside the material can be obtained.

The combination of OCT techniques with microscopic methods and using 2D CCD or CMOS cameras instead of point detectors as imaging device results in full-field (FF) coherent probe microscopic (CPM) imaging [2, obtaining information about both axial and lateral sample structures, with decoupled depth and lateral resolutions.

Although OCT was originally developed for the biomedical diagnostics, recently, OCT techniques have shown their potential in the field of material research 35]. In a similar way, also for CPM as full-field imaging technique a comparable tendency focusing on material research can be noted [4. Exploiting CPM imaging for micro-material inspection represents the aim in our experimental optical configuration. We describe the challenges for routinely evaluating measured CPM scans and suggest in the following a scheme for automated image analysis starting from the demodulation of the raw data, the enhancement of the resulting demodulated images, tested methods for orientation estimation of internal fiber structures, finally allowing a clustering of structures or image slices.

\section{Methods}

\subsection{CPM Measurement Setup and Materials}

A scheme of our CPM optical setup is shown in Fig. 1. depicted here in a Michelson configuration [2]. As low-coherence light source we apply likewise a superluminiscent diode (Superlum, central wavelength $=850 \mathrm{~nm}$, spectral bandwidth $=50 \mathrm{~nm}$ ) and a super-continuum light source (LEUKOS SM-30, central wavelength $=825 \mathrm{~nm}$, spectral bandwidth $=350 \mathrm{~nm}$ ), which provide an axial resolution of about $16 \mu \mathrm{m}$ and $2 \mu \mathrm{m}$ in air, respectively. The lateral resolution, determined by the focusing optical components totals about $3 \mu \mathrm{m}$. The achievable depth range for imaging yields about 200-500 $\mu \mathrm{m}$ dependent on the investigated material and the utilized wavelength range. Our conventional CPM system can be extended additionally by the possibilities as given in microscopy, e.g. by the modification of the applied imaging contrast [6]. In this paper we demonstrate an application for micro-material imaging using brightfield contrast.

In particular, polymers reinforced with fiber micro-structures are the technical materials in the scope of our interest. They contain internal (glass) fiber components, which may be randomly distributed, but which are often arranged 


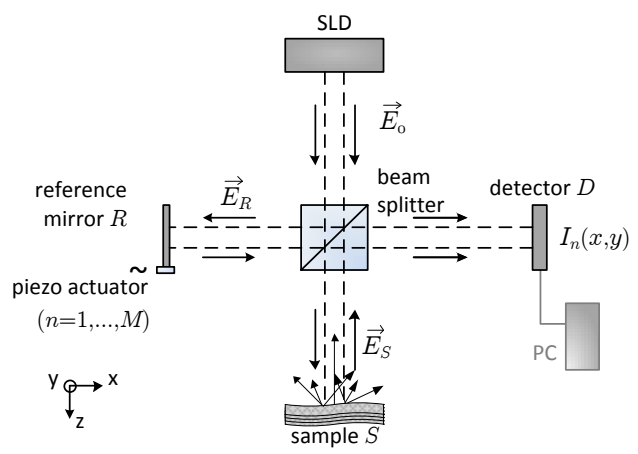

Fig. 1. Scheme of the Coherence Probe Microscopy optical setup, here illustrated in a Michelson configuration. The imaging lens systems are neglected in the drawing for sake of simplicity.

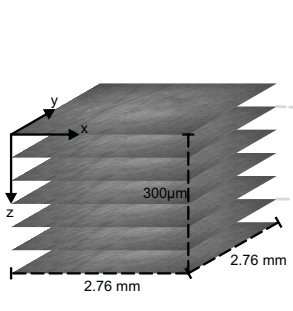

(a)

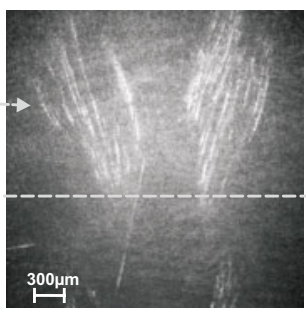

(b)

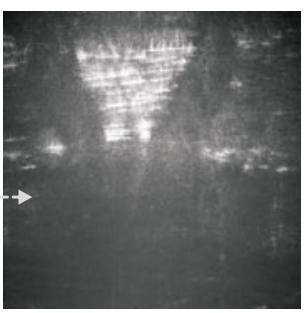

(c)

Fig. 2. Illustration for CPM imaging samples: (a) sketch of the CPM image stack taken as en-face scans $(x, y)$ over depth $z ;(\mathrm{b})$ and (c) show two (already demodulated) CPM slices (amplitude images) recorded at different imaging depths, taken from fiber structures casted in a polymer matrix. The depth varying orientation of the fiber structures is clearly visible in this sample: the almost diagonal fiber structures in (b) change to horizontal internal structures in (c).

just as in a layered configuration. The fibers can be knitted or are appearing in a woven structure. Different fiber layers may be discerned over depth by their distinguished orientational characteristics of internal structures, as illustrated in Fig. 2. Additionally to the intended and designed fiber distribution the resulting CPM image stack may also contain scans showing typical artifacts, so called 'ghost-images'. These erroneous structures may occur due to multiple reflections at interfaces or are caused by the possible side-lobes of the autocorrelation function of the light source. These erroneous slices are representing outliers. As outliers complicate any analysis they should be automatically detected.

\subsection{Image Demodulation}

At each depth position $z$ a sequence of interferometric images is taken in a phaseshifted way by equally-spaced phase steps $\phi_{M}(x, y)$ (in our case, with $M$ up to 
8 frames) between subsequent frames $I_{M}(x, y)$. These interferometric raw data images have to be demodulated, applying here a complex vector addition scheme 6

$$
I_{C}(x, y)=\sum_{M} I_{M}(x, y) \exp \left(-i \phi_{M}(x, y)\right)
$$

delivering the amplitude image $I(x, y)=a b s\left(I_{C}(x, y)\right)$ and the phase image $\phi(x, y)=\arg \left(I_{C}(x, y)\right)$. The thereby obtained amplitude images $I(x, y)$, as shown in Fig. 2, are used in the following for the demonstrated image analysis as they contain the structural information of interest. (It should be mentioned, that the amplitude information could be extended by analyzing the phase image too. However, these phase-based approaches require extended unwrapping procedure [7.)

The final sample images exhibit a field of view of $2.758 \times 2.758 \mathrm{~mm}$ and the stepwidth into the depth is $3 \mu \mathrm{m}$. The example image stack counts 100 slices.

\subsection{Image Enhancement}

CPM scans, as taken by an interferometric imaging technique, are often highly disturbed by speckles. These speckles exhibit a two-fold nature: on one-hand they describe a noise component of the signal, but on the other hand they carry information about the internal structure. This ambiguity and the scattering behavior of the micro-structures result that the imaged features often appear in a patchy, non-continuous way, although the real structures are almost continuous. Furthermore, a non-uniform background illumination, fluctuations during the phase-stepping, and multiple scattering or reflections complicate an automatic analysis. Therefore, we have tested different denoising and background correction techniques to enhance the quality of the primary CPM-images.

Denoising. Discussing noise in the measured image $I_{n}(x, y)$, the speckle noise $n(x, y)$ has to be regarded as the dominant contribution, degrading the theoretical CPM image $I(x, y)$. Speckle noise (related to intensity) can be modeled in a multiplicative way. However, in the OCT community mostly a logarithm transform is applied on the image which converts the speckle noise into an additive component, $\log \left(I_{n}(x, y)\right)=\log (I(x, y))+\log (n(x, y))$. This logarithm scaled image is taken by us as basis for our further considerations.

As a well-established method for reducing speckle noise, different adaptive median filtering techniques are mentioned in literature 8]. Additionally, we have investigated a denoising approach based on the curvelet transform as introduced originally by Candes et. al. in [9]. For our application, it shows the advantage that the elongated fiber structure are taken into account. These fiber structures are then enhanced, whereas the randomly and isotropically distributed speckle noise is removed.

As third image enhancement technique tested a denoising based on the á trous wavelet transform [10] is performed. The á trous wavelet transform applies a, by a factor of 2 , increasingly sized scaling function. At each scale the holes within the up-sampled scaling function are filled by zero values. It may be realized as a 
fast discrete wavelet transform by subsequently performing a low pass filtering in a convolutive way, being in particular of interest for the processing of the huge amount of data. In our case, we have taken a $B_{3}$-spline scaling function as convolution mask for our images.

Background Correction. Due to the collimated illumination the background exhibits a radial illumination profile which hampers the following analysis. Therefore, a further image enhancement step comprehends a correction for the non-uniformly shaped background. We have used both, a method based on a morphological opening approach, the well-established so-called rolling ball algorithm as introduced by Sternberg [11, and a method exploiting an (only) phasebased reconstruction scheme applying monogenic wavelets for an equalization of brightness, as recently suggested in 12 .

\subsection{Orientation Estimation}

After preprocessing of the images with different enhancement techniques we are now able to analyze and to characterize the inner structure of the samples, where especially the fiber structure orientation indicating the different fiber layers, should be considered. We have focused on structure tensor methods and in addition on, complex wavelet-based methods as Gabor filtering and a monogenic wavelet-based orientation estimation.

Structure Tensor-based Orientation Estimation. As a first approach to get information about orientation and isotropy properties on internal fiber structures within the region of interest we have applied a structure tensor-based orientation estimation, which is based on the minimal deviation gradient direction 13

$$
\theta\left(\mathbf{x}_{0}\right)=\arg \max _{\theta \in[-\pi, \pi]} \int_{\mathbb{R}^{2}} w\left(\mathbf{x}-\mathbf{x}_{0}\right)\left|\nabla f(\mathbf{x})^{T} \overline{\mathbf{n}}\right|^{2} d \mathbf{x} .
$$

Here, $w(\mathbf{x}) \geq 0$ is a weighting function that specifies the area of interest and $\overline{\mathbf{n}}$ represents the local orientation $\overline{\mathbf{n}}=[\cos \theta \sin \theta]^{T}$. Rewriting the right-hand side of equation (2) a tensor form is given by

$$
\int_{\mathbb{R}^{2}} w\left(\mathbf{x}-\mathbf{x}_{0}\right)\left|\nabla f(\mathbf{x})^{T} \overline{\mathbf{n}}\right|^{2} d \mathbf{x}=[\cos \theta \sin \theta] \mathbf{J}\left(\mathbf{x}_{0}\right)\left[\begin{array}{c}
\cos \theta \\
\sin \theta
\end{array}\right] .
$$

With the help of the structure tensor $\mathbf{J}=\left(\begin{array}{c}J_{11} J_{12} \\ J_{12} J_{22}\end{array}\right)=\langle\nabla f(\mathbf{x}) \otimes \nabla f(\mathbf{x})\rangle_{w}$, determined by the weighted outer product $\left(\langle\otimes\rangle_{w}\right)$ of the image gradient $\nabla f(\mathbf{x})=$ $\nabla I(x, y)$, the orientation angle $\theta$

$$
\theta=\frac{1}{2} \arctan \left(\frac{2\left(J_{12}\right)}{\left(J_{22}\right)-\left(J_{11}\right)}\right)
$$


the coherence $\chi$, as measure for the isotropy of the pattern in the local neighborhood,

$$
\chi=\frac{\sqrt{\left(\left(J_{22}\right)-\left(J_{11}\right)\right)^{2}+4\left(J_{12}\right)^{2}}}{\left(J_{11}\right)+\left(J_{22}\right)},
$$

and the energy $E$, as measure for significance,

$$
E=\operatorname{trace}(\mathbf{J})=\left(J_{11}\right)+\left(J_{22}\right),
$$

can be defined.

Orientation Estimation by the Gabor Wavelet Transform. In contrast to the structure tensor approach, the Gabor wavelet transform 14 as a linear filter, may be described by its complex-valued filter function $g(x, y)=w_{r}(x, y)$. $s(x, y)$, being a 2D Gaussian kernel function $w_{r}(x, y)$ modulated by a complex exponential plane wave $s(x, y)$. The filter applied in different orientations $\theta$ gives highest response in the orientation best fitting to the inner structure of the image.

Monogenic Wavelet-based Orientation Estimation. As a third approach for the local orientation estimation of internal structures we tested a monogenic wavelet-based method for the structure tensor following an approach as described in [15. In analogy to equation (2), by replacing the directional derivative with the directional Hilbert operator $\mathcal{H}_{\theta}$ and the gradient by the Riesz operator $\mathcal{R}$, as defined in [1516, now the function

$$
\theta\left(\mathbf{x}_{0}\right)=\arg \max _{\theta \in[-\pi, \pi]} \int_{\mathbb{R}^{2}} w\left(\mathbf{x}-\mathbf{x}_{0}\right)\left|\mathcal{H}_{\theta} f(\mathbf{x})\right|^{2} d \mathbf{x}
$$

is maximized over a local neighborhood specified by the weighting function $w(\mathbf{x})=w(-\mathbf{x}) \geq 0$. The right-hand side of equation (7) can be rewritten in tensor form by

$$
\int_{\mathbb{R}^{2}} w\left(\mathbf{x}-\mathbf{x}_{0}\right)\left|\mathcal{H}_{\theta} f(\mathbf{x})\right|^{2} d \mathbf{x}=[\cos \theta \sin \theta] \mathbf{J}\left(\mathbf{x}_{0}\right)\left[\begin{array}{c}
\cos \theta \\
\sin \theta
\end{array}\right],
$$

where $\mathbf{J}\left(\mathbf{x}_{0}\right)$ now contains the directional Hilbert transforms (HT) of $f(\mathbf{x})$ as components similar to equation (3). Additionally, this optimization is performed on different scalings, given by Laplacian like spline wavelet basis. At scale $i$ a wavelet-based structure tensor is expressed by

$$
\left[\mathbf{J}_{i}(\mathbf{k})\right]_{m n}=\sum_{\mathbf{l} \in \mathbb{Z}^{2}} w[\mathbf{l}-\mathbf{k}] r_{m, i}[\mathbf{l}] r_{n, i}[\mathbf{l}]
$$

with $m, n \in\{1,2\}$, for a given weighting sequence $w[\mathbf{l}] \geq 0$. The term $r_{1, i}[\mathbf{l}]$ represents the real and $r_{2, i}[\mathbf{l}]$ the imaginary part of the complex-valued wavelet coefficients at scale $i$ in a monogenic wavelet basis, obtained by applying the Riesz resp. directional HT on an isotropic wavelet basis, as suggested in [15. 


\section{Results}

\subsection{Image Enhancement}

We have exemplified the described image analysis procedure on several fiberreinforced polymer samples investigated by our CPM optical setup. The polymer matrix of these samples consisted of either epoxy resine, poly-propylene or polyethylene material, whereas the reinforcement mainly was given by glass-fibers, included in preferred directions or in a random manner within the polymer material. We show our results for one typical representative of these glass-fiber reinforced polymer samples, containing glass-fibers embedded in an epoxy resin with different orientation layers. The radial background illumination can clearly be recognized in Fig. 3 (a) and is emphasized in the corresponding binary version (b). As shown in Fig. 3 (c) and (d), the background is sufficiently removed by the morphological correction, whereas the correction given by equalization of brightness is depicted in Fig. 3 (e) and (f). For our purposes morphological background correction proves most suitable, as so-called ghost structures (multi-reflections) are better suppressed. The different denoising results are illustrated in Fig. 4. Whereas median filtering gives insufficient results, as visible in Fig. 4 (a) and (b), the curvelet-based denoising fits well for the fiber structures, as shown Fig. 4 (c) and (d). Here, the á trous wavelet-based method is combined with a corresponding masking and segmentation, as depicted in Fig. 4 (e) and (f).
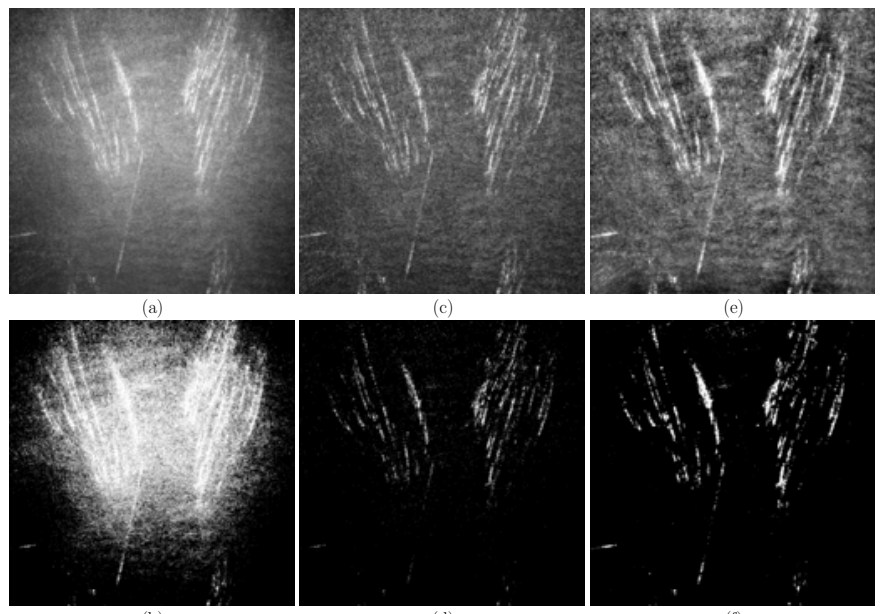

(d)

(f)

Fig. 3. Background correction exemplified on the fiber-reinforced polymer: (a) the demodulated amplitude image as used for analysis, applying in (c) the morphological correction method and (e) the monogenic wavelet-based equalization of brightness approach [12. The corresponding binary versions are shown in (b), (d), and (f). As clearly recognizable, the originally radial illumination profile could be removed. 

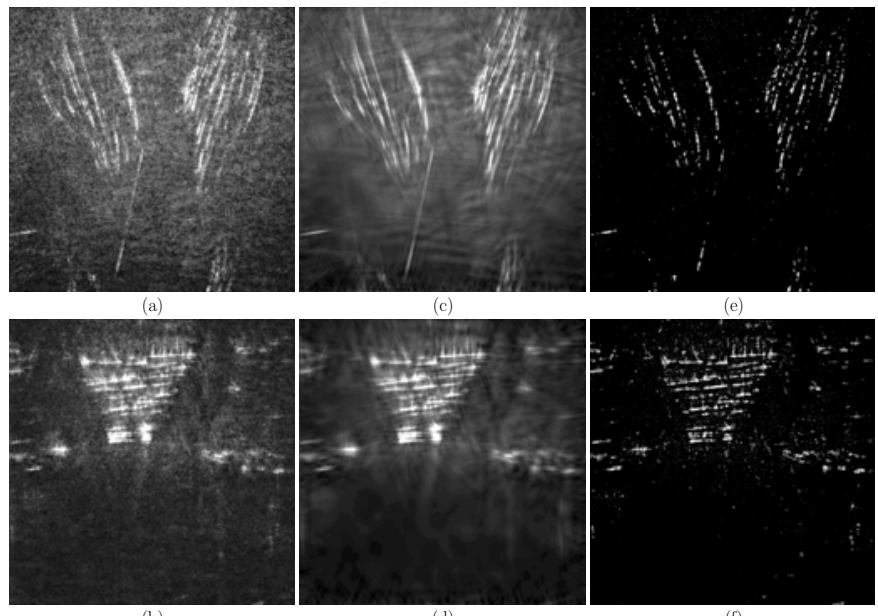

(d)

(f)

Fig. 4. Denoising illustrated for the CPM slices as depicted in Fig. 2 (b) and (c): applying (a) and (b) median filtering, (c) and (d) curvelet-based denoising, (e) and (f) á trous wavelet-based denoising method

\subsection{Orientation Estimation}

The local orientation values for fiber structures given by the different estimation methods are comparatively depicted in Fig. 5, where the original image is enhanced by the median denoising method and the rolling ball background correction. The conventional structure tensor-based orientation estimation (a) is clearly outperformed by both the Gabor wavelet (b) and the monogenic waveletbased method (c). The latter has shown to be more robust to noisy structures due to its multi-scale character, partly allowing to skip an extensive denoising before. Also the entropy of the orienation in Fig. 5 (b) is smaller than the one of (a). Therefore, we have performed our cluster analysis, on features obtained by this monogenic wavelet-based estimation scheme.

Having evaluated the different pre-processing and orientation estimation methods on the investigated polymer samples and additionally validated by simulations we finally suggest the following processing scheme: A) Background estimation by the rolling ball algorithm, B) Denoising by the median filter (or can be ommitted), and C) Orientation estimation by the monogenic wavelet-based method.

\subsection{Clustering}

As final step towards an automatic classification k-means clustering has been performed. First, the clustering is applied on a data set of feature vectors, having the computed local orientation, coherency, and energy, defined in each pixel, as their entries. Here, the initial clustering is used for classifying the internal fiber 

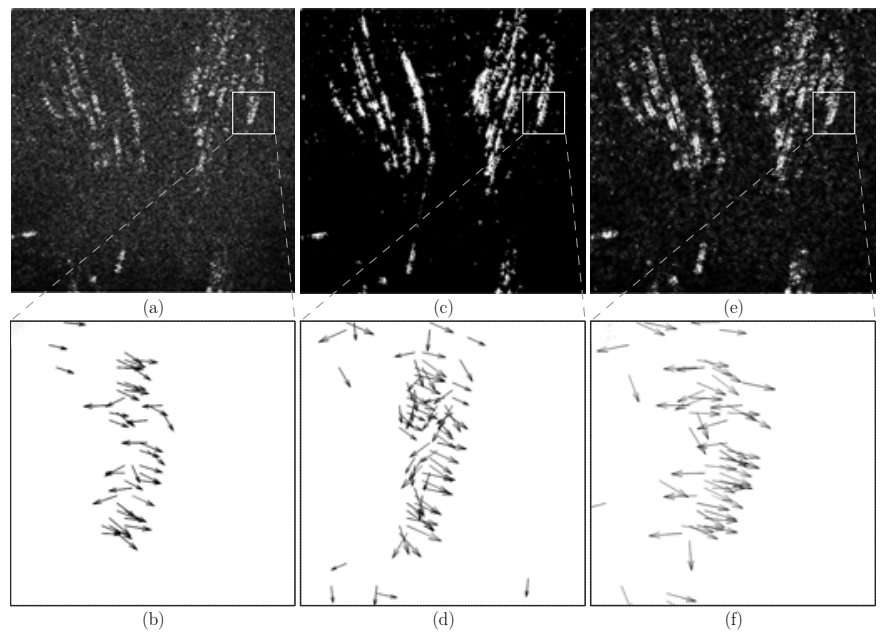

Fig. 5. Local orientation estimation for the enhanced internal fiber structures, performed in (a) and (b) with the conventional structure tensor approach, in (c) and (d) with a Gabor filter-based method, (e) and (f) with a modified structure tensor approach exploiting monogenic wavelets. The usual HSV representation (with hue: orientation, saturation: coherency, brightness: energy according to equations (44),(5) and (6)), is replaced here by a vector plot for a better visibility in gray-scale coding.
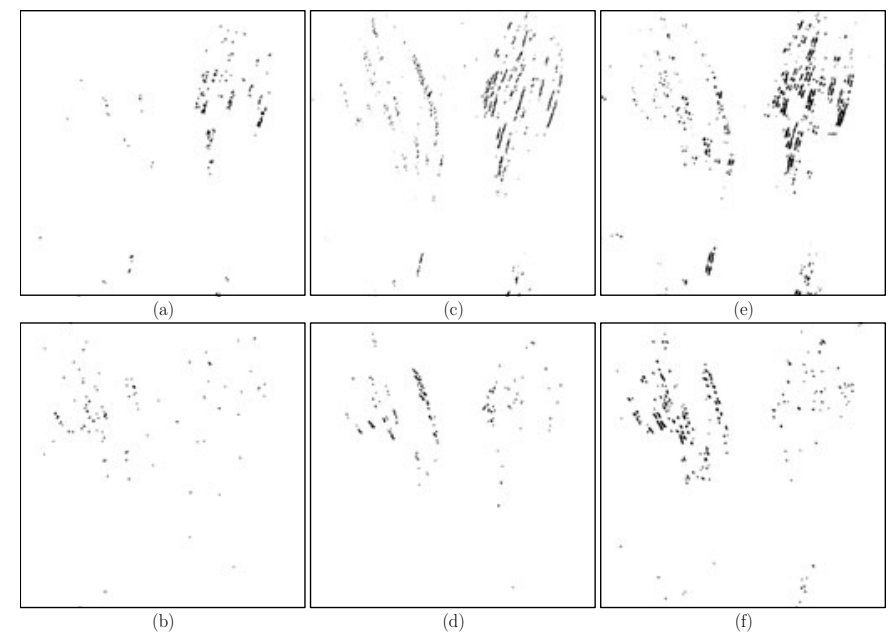

Fig. 6. Clustering of the internal fiber structures within a single slice (here into three classes: The black area in the images shows the pixels belonging to a class. The top images (a), (c) and (e) are showing the first class, the bottom images (b), (d) and (f) are showing the second class. The third class are the remaining pixel points, the background. The k-means clustering was applied on data (features concerned: local orientation, coherency, and energy) obtained by: (a) and (b) the (conventional) structure tensor-based estimation, (c) and (d) the Gabor wavelet-based method, (e) and (f) the monogenic wavelet-based method, [15]. 

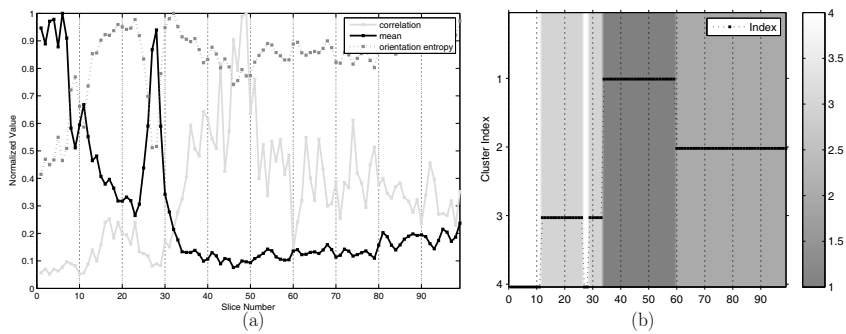

Fig. 7. Clustering of slices within the whole CPM data stack: (a) representation of slice-related normalized features (cross-correlation (gray), mean intensity value (black), entropy of the orientation distribution (dotted)) over depth, (b) resulting clusters, where surface/outliers (white), diagonal (lightgray) and horizontal (darkgray) fiber slices, and fiber layer blendings (medium gray) are automatically discernible

structures within a single slice into three classes, as depicted in Fig. 6. The visibility of the results was improved by a median filter and an morphologic open operation.

Additionally, a second clustering is realized over the whole CPM data stack for classifying all slices into four different categories: slices containing fiber layers of distinguished principal orientations, into surface region, and into erroneous 'ghost images'. In contrast to Fig. 6, which is a pixel based clustering, the clustering in Fig 7 is performed slicewise. The normalized cross-correlation between subsequent scans, the mean value, and entropy of the orientation distribution, defined for each slice, give the entries for the feature vector here. These features calculated for each slice, as depicted in Fig. 7 (a), were taken for the clustering. The resulting clusters, into which the subsequent slices may by grouped, are depicted in Fig. 7 (b). Clearly recognizable in the clustering are the distinguished fiber orientations layers (light and dark gray), the outliers and the surface (white both, as outliers are weak repetitions of the surface here), and the deeper bulk material, where no sufficient information can be gained (medium gray).

\section{Conclusions}

Summarizing we have shown a complete imaging and image analysis procedure suitable for the investigation of fiber-reinforced polymers. In conclusion, we have demonstrated the capability of our established CPM setup and the potential, which can be achieved in combination with image processing tools for a better visualization, analysis and classification of the internal fiber structures within technical materials, finally enabling an automatized evaluation of CPM image data.

Acknowledgments. The financial support by the Federal Ministry of Economy, Family and Youth and the National Foundation for Research, Technology and Development is gratefully acknowledged. Furthermore, we thank Katja Schladitz at the Fraunhofer Institute ITWM, Kaiserslautern for fruitful discussions and 
providing the MAVI 3D image processing software. For computing the monogenic orientation and equalization of brightness we have applied both wavelet toolboxes: MonogenicJ [15] and Monogenic Wavelet Toolbox [12].

\section{References}

1. Drexler, W., Fujimoto, J.G.: Optical coherence tomography. Springer, Berlin (2008)

2. Dubois, A., Vabre, L., Boccara, A., Beaurepaire, E.: High-resolution full-field optical coherence tomography with a Linnik microscope. Appl. Opt. 41, 805-812 (2002)

3. Stifter, D.: Beyond biomedicine: a review of alternative applications and developments for optical coherence tomography. Appl. Phys. 88, 337-357 (2007)

4. Latour, G., Echard, J.P., Soulier, B., Emond, I., Vaiedelich, S., Elias, M.: Structural and optical properties of wood and wood finishes studied using optical coherence tomography. Appl. Optics 48, 6485-6491 (2009)

5. Wiesauer, K., Pircher, M., Gotzinger, E., Hitzenberger, C.K., Engelke, R., Ahrens, G., Gruetzner, G., Stifter, D.: Transversal ultrahigh-resolution polarizationsensitive optical coherence tomography for strain mapping in materials. Optics Express 14, 5945-5953 (2006)

6. Schausberger, S.E., Heise, B., Maurer, C., Bernet, S., Ritsch-Marte, M., Stifter, D.: Flexible contrast for low-coherence interference microscopy by Fourier-plane filtering with a SLM. Opt. Lett. 35, 4154-4156 (2010)

7. Stifter, D., Leiss-Holzinger, E., Major, Z., Baumann, B., Pircher, M., Götzinger, E., Hitzenberger, C.K., Heise, B.: Dynamic optical studies in materials testing with spectral-domain polarization-sensitive OCT. Opt. Express 18, 25712-25725 (2010)

8. Gallagher, N.C., Wise, G.L.: Median filters: a tutorial. In: Proc. IEEE ISCAS, vol. 88, pp. 1737-1744 (1988)

9. Candes, E.J., Demanet, L., Donoho, D.L., Ying, L.: Fast Discrete Curvelet Transform (2005)

10. Shensa, M.J.: The discrete wavelet transform: Wedding the Á trous and Mallat algorithms. IEEE Trans. Signal Proc. 40, 2464-2482 (1992)

11. Sternberg, S.R.: Biomedical image processing. Computer 16, 22-34 (1983)

12. Held, S., Storath, M., Massopust, P., Forster, B.: Steerable wavelet frames based on the Riesz transform. IEEE Trans. Image Proc. 19, 653-667 (2010); Monogenic wavelet toolbox, http://www.mamebia.de/Software

13. Jähne, B.: Digital Image Processing. Springer, Heidelberg (2002)

14. Movellan, J.R.: Tutorial on Gabor Filters

15. Unser, M., Sage, D., Ville, D.V.D.: Multiresolution monogenic signal analysis using the Riesz-Laplace wavelet transform. IEEE Trans. Image Proc. 18, 2402-2418 (2009); MonogenicJ toolbox, http://bigwww.epfl.ch/demo/monogenic/

16. Felsberg, M., Sommer, G.: The monogenic signal. IEEE Trans. Image Proc. 49, $3136-3144$ (2001) 